math.NT/0105120

\title{
Sur certains espaces de Hilbert de fonctions entières, liés à la transformation de Fourier et aux fonctions $\mathrm{L}$ de Dirichlet et de Riemann
}

\author{
Jean-François BURNOL
}

Mai 2001

\begin{abstract}
Nous construisons dans un Espace de Sonine de fonctions entières un sous-espace attaché à la fonction dzêta de Riemann et nous montrons que le quotient contient des vecteurs intrinsèquement liés aux zéros non-triviaux et à leurs multiplicités éventuelles.
\end{abstract}

Université de Nice Sophia-Antipolis

Laboratoire J. A. Dieudonné, Mathématiques

Parc Valrose

F-06108 Nice cedex 02

France

burnol@math.unice.fr 


\section{English summary}

Let $K=L^{2}((0, \infty), d t)$. The Mellin transform of a function $f \in K$ is defined, on the critical line $\operatorname{Re}(s)=1 / 2$ and with the meaning of a Plancherel isometry, through the formula $\widehat{f}(s)=$ $\int_{0}^{\infty} f(t) t^{s-1} d t$. Let $\mathcal{F}_{+}$be the cosine transform (formally $\mathcal{F}_{+}(f)(t)=2 \int_{0}^{\infty} \cos (2 \pi t u) f(u) d u$.) Let $a$ and $b$ be two strictly positive real numbers and let $K_{a, b}$ be the closed subspace of $K$ consisting of functions $f$ with support in $(a, \infty)$ and such that $\mathcal{F}_{+}(f)$ has its support in $(b, \infty)$. It is known classically that $L^{2}((0, a])+\mathcal{F}_{+}\left(L^{2}((0, b])\right)$ is a closed proper subspace of $K$ (see [15], sect. 2.9, $p$. 126-127) hence that none of the $K_{a, b}$ 's is reduced to $\{0\}$. The functions $\pi^{-(1-s) / 2} \Gamma((1-s) / 2) \widehat{f}(s)$ on the critical line (for $f \in K_{a, b}$ ) build (up to a trivial change of variable) a special case of a Sonine Hilbert space as considered by De Branges in [13], and in particular it consists of entire functions. We prove elementarily:

THÉORÈme 1. The Mellin transforms of the functions in $K_{a, b}$ are entire functions. They have at $s=1+2 j, j \in \mathbb{N}$, trivial zeros and these are their only common zeros (and are simple as such.)

We write $(\phi, \psi]$ for the euclidean scalar product $\int \phi(u) \psi(u) d u$. Let $I$ be the operator $I(f)(t)=$ $1 / t f(1 / t)$ and let $\mathcal{G}=I \mathcal{F}_{+} I$. The operator $\mathcal{G}$ is unitary and satisfies $\mathcal{G}^{2}=1$. Let $\Lambda>0$ and let $H_{\Lambda}=I\left(K_{1 / \Lambda, 1 / \Lambda}\right)=L^{2}((0, \Lambda), d t) \bigcap \mathcal{G}\left(L^{2}((0, \Lambda), d t)\right)$. The Mellin transforms of elements from $H_{\Lambda}$ are entire functions with trivial zeros at $-2 j, j \in \mathbb{N}$. The function $\widehat{f}(s)$ satisfies the same functional equation as the Riemann zeta function if and only if $\mathcal{G}(f)=f$, and up to a sign if and only if $\mathcal{G}(f)=-f$. We prove:

Theorem 2. One has $\bigcap H_{\Lambda}=\{0\}$ and $\overline{\bigcup H_{\Lambda}}=K$. For each $\Lambda>0$, each complex number $w$, and each integer $k \in \mathbb{N}$ there is a unique vector $Z_{w, k}^{\Lambda}$ in $H_{\Lambda}$ such that the euclidean scalar product $\left(f, Z_{w, k}^{\Lambda}\right]$ equals $\left(d^{k} / d^{k} w\right)\left(\pi^{-w / 2} \Gamma(w / 2) \widehat{f}(w)\right)$ for each $f \in H_{\Lambda}$. The vectors $Z_{w, k}^{\Lambda}, w \in \mathbb{C}, k \in \mathbb{N}$ are linearly independent, in particular they are all non-vanishing.

Let $\Lambda>1$ and let $\mathcal{V}_{\Lambda}$ be the vector space of smooth functions with support in $[1 / \Lambda, \Lambda]$. Let $D$ be the operator $u\left(d^{2} / d^{2} u\right) u$. Let, for $\phi \in \mathcal{V}_{\Lambda}, E(\phi)(u)=\sum_{n \geq 1} \phi(n u)-\left(\int_{0}^{\infty} \phi(t) d t\right) / u$.

Theorem 3. Let $W_{\Lambda}$ be the closure in $K$ of the functions $E(\phi)$ for $\phi \in D\left(\mathcal{V}_{\Lambda}\right)$. One has 
$W_{\Lambda} \subset H_{\Lambda} . A$ vector $Z_{w, k}^{\Lambda}$ is perpendicular to $W_{\Lambda}$ if and only if $w$ is a non-trivial zero $\rho$ of the zeta function of Riemann and $0 \leq k<m_{\rho}\left(m_{\rho}=\right.$ multiplicity of $\rho$.)

The outlines of the proofs will be found in the french part of this Note. One thus defines $H P_{\Lambda}$ as the perpendicular complement to $W_{\Lambda}$ in $H_{\Lambda}$ so that

$$
K=\left(L^{2}((\Lambda, \infty), d t)+\mathcal{G} L^{2}((\Lambda, \infty), d t)\right) \perp W_{\Lambda} \perp H P_{\Lambda}
$$

The construction should be compared to the approach of Connes in [11], [12].

Conclusion. In the continuation of [4], [5], we have shown that the scattering theory for the Fourier transform (which belongs to Analysis) has arithmetical aspects, through exhibiting some natural Hilbert spaces containing vectors intrinsically associated to the non-trivial zeros of the Riemann zeta function (and to their multiplicities; this is a continuation to [10].) It would be interesting to better understand the operator framework behind this construction $([6],[7],[8]$.$) The$ study of $\Lambda \rightarrow 0, \Lambda=1, \Lambda \rightarrow \infty$ is a problem of the renormalization group ([16]), which appears to be linked to the problem of the nature of the zeta function ([3].)

Dans la section intitulée "Espaces de Sonine" de son livre ([13]) De Branges associe des espaces de Hilbert de fonctions entières à la transformation de Hankel d'ordre $\nu, \nu>-1$. Lorsque $\nu=-1 / 2$ la transformation de Hankel est essentiellement la transformation en cosinus $\mathcal{F}_{+}$agissant sur $K=$ $L^{2}(] 0, \infty[, d t)$ selon (formellement) $\mathcal{F}_{+}(f)(t)=2 \int_{0}^{\infty} \cos (2 \pi t u) f(u) d u$. Nous construisons dans la présente Note des quotients de ces Espaces de Sonine (associés à $\mathcal{F}_{+}$) qui contiennent des vecteurs intrinsèquement liés aux zéros non-triviaux de la fonction dzêta de Riemann (et à leurs multiplicités éventuelles.) Une description plus satisfaisante de cette construction est de nature opératorielle, et nous reviendrons sur ce point dans un travail ultérieur.

\section{Les espaces $K_{a, b}$}

Soit $K$ l'espace de Hilbert $L^{2}(] 0, \infty[, d t)$ des fonctions à valeurs complexes de carrés intégrables sur ] $0, \infty\left[\right.$. Nous noterons $(\phi, \psi]$ pour le produit scalaire euclidien $\int \phi(u) \psi(u) d u$. Soit $\mathcal{F}_{+}$la transformation en cosinus agissant formellement selon $\mathcal{F}_{+}(f)(t)=2 \int_{0}^{\infty} \cos (2 \pi t u) f(u) d u$. Elle vérifie $\mathcal{F}_{+}^{2}=1$, 
$\left(\mathcal{F}_{+}(\phi), \mathcal{F}_{+}(\psi)\right]=(\phi, \psi]$. Soit $I$ agissant selon $I(f)(t)=1 / t f(1 / t)$. Le composé $\Gamma_{+}=\mathcal{F}_{+} I$ est invariant et est donc diagonalisé par l'isométrie de Mellin-Plancherel $f \mapsto \widehat{f}(s)=\int f(t) t^{s-1} d t$ entre $K$ et l'espace des fonctions de carrés intégrables sur $\operatorname{Re}(s)=1 / 2$ pour la mesure $|d s| / 2 \pi$. Le multiplicateur spectral associé est la fonction $\gamma_{+}(s)=2(2 \pi)^{-s} \cos (\pi s / 2) \Gamma(s)$. On a donc $\gamma_{+}(s) \gamma_{+}(1-s)=1$ et, presque partout sur la droite critique:

$$
\forall f \in K \quad \widehat{f}(s)=\gamma_{+}(s) \widehat{\mathcal{F}_{+}(f)}(1-s)
$$

Soient $a$ et $b$ deux nombres réels strictement positifs et considérons le sous-espace fermé $K_{a, b}$ de $K$ des fonctions $f$ supportées par $\left[a, \infty\left[\right.\right.$ et telles que $\mathcal{F}_{+}(f)$ soit supportée par $[b, \infty[$. Il est connu classiquement que $\left.\left.L^{2}([0, a])+\mathcal{F}_{+}\left(L^{2}(] 0, b\right]\right)\right)$ est un sous espace fermé propre de $K$ (voir [15], sect. 2.9, p. 126-127) et donc que les espaces $K_{a, b}$ sont tous non-réduits à $\{0\}$. Si $f$ est presque partout nulle sur $] 0, a]$ alors $\int f(t) t^{s-1} d t$ est analytique dans le demi-plan $\operatorname{Re}(s)<1 / 2$, et par $(1.1)$ si $f \in K_{a, b}$ alors $\widehat{f}(s)$ existe en tant que fonction analytique dans $\operatorname{Re}(s)>1 / 2$. En fait:

THÉORÈme 1.1. (voir [13]) Toute fonction d'un espace $K_{a, b}$ a une transformée de Mellin qui est une fonction entière de $s \in \mathbb{C}$.

Il suffirait de constater en effet que les fonctions $\pi^{-(1-s) / 2} \Gamma((1-s) / 2) \widehat{f}(s)$ forment (à un changement de variables près) un des Espaces de Sonine de fonctions entières considérés par De Branges dans [13]. Mais pour établir en particulier le Théorème (1.5) plus loin, nous ferons plutôt reposer la démonstration sur l'identité élémentaire suivante, valable pour tout $f \in K_{a, b}$ :

$$
\int_{0}^{\infty} f(t) t^{s-1} d t=\int_{b}^{\infty} \frac{(1-s) \int_{a}^{\infty} \sin (2 \pi u t) t^{s-2} d t-a^{s-1} \sin (2 \pi a u)}{\pi u} \mathcal{F}_{+}(f)(u) d u
$$

Cette identité est tout d'abord établie pour $\operatorname{Re}(s)<0$ et donne le prolongement analytique de $\widehat{f}(s)$ au moins au demi-plan $\operatorname{Re}(s)<1$. Puis (par exemple) (1.1) donne le prolongement analytique au plan complexe. L'équation (1.2) résulte du calcul (immédiat) par intégration par parties:

$$
\operatorname{Re}(w)<0 \Rightarrow 2 \int_{a}^{\infty} \cos (2 \pi u t) t^{w-1} d t=\frac{(1-w) \int_{a}^{\infty} \sin (2 \pi u t) t^{w-2} d t-a^{w-1} \sin (2 \pi a u)}{\pi u}
$$

Avec $C_{a}(u, w)=2 \int_{a}^{\infty} \cos (2 \pi u t) t^{w-1} d t$ et $S_{a}(u, w)=2 \int_{a}^{\infty} \sin (2 \pi u t) t^{w-1} d t$ on a la relation ci-dessus entre $C_{a}(u, w)$ et $S_{a}(u, w-1)$ et aussi une relation entre $S_{a}(u, w)$ et $C_{a}(u, w-1)$ qui établissent que ces fonctions sont des fonctions entières de $w$ (pour chaque $u>0$ fixé) et aussi 
qu'elles sont $O(1 / u)$ sur $[1, \infty[$ uniformément par rapport à $w \in \mathbb{C}$ lorsque $|w|$ est borné. L'usage que nous ferons de ce dernier point est dans la continuation de [10] (motivé par [2], Lemme 6.) Il va sans dire que ces calculs simples (et ceux qui suivent) sont bien connus.

Soit $f(t)$ une fonction infiniment dérivable à support compact dans $] 0, \infty[$. Par Fubini on a $\int_{a}^{\infty} \mathcal{F}_{+}(f)(t) t^{w-1} d t=\int_{0}^{\infty} C_{a}(u, w) f(u) d u$ pour $\operatorname{Re}(w)<0$ puis pour tout $w \in \mathbb{C}$ puisque les deux membres sont des fonctions entières de $w$. Soit $D_{a}(u, w)=2 \int_{0}^{a} \cos (2 \pi u t) t^{w-1} d t(\operatorname{pour} \operatorname{Re}(w)>0$.) Par Fubini on a $\int_{0}^{a} \mathcal{F}_{+}(f)(t) t^{w-1} d t=\int_{0}^{\infty} D_{a}(u, w) f(u) d u$ et donc $\left.\widehat{\mathcal{F}_{+}(f}\right)(w)=\int_{0}^{\infty}\left(C_{a}(u, w)+\right.$ $\left.D_{a}(u, w)\right) f(u) d u$ d'où par (1.1) pour $\operatorname{Re}(w)>0$ :

$$
C_{a}(u, w)=\gamma_{+}(w) u^{-w}-2 \int_{0}^{a} \cos (2 \pi u t) t^{w-1} d t=\gamma_{+}(w) u^{-w}-2 \sum_{j=0}^{\infty} \frac{(-1)^{j}}{(2 j) !}(2 \pi u)^{2 j} \frac{a^{2 j+w}}{2 j+w}
$$

Cette dernière formule montre que $C_{a}(u, w)$ pour $w$ fixé est une fonction analytique de $u$ dans $\mathbb{C} \backslash]-\infty, 0]$, au moins pour $w$ distinct de $0,-2,-4, \ldots$ En utilisant le fait que $\gamma_{+}(w)$ a un pôle simple en $-2 k(k \in \mathbb{N})$ avec un résidu égal à $2(2 \pi)^{2 k}(-1)^{k}(1 /(2 k) !)$, on voit de plus que $C_{a}(u,-2 k)$ diffère d'une fonction entière de $u$ par un multiple non nul de $u^{2 k} \log (u)$ et qu'elle est donc également analytique en $u$ sur $\mathbb{C} \backslash]-\infty, 0]$. On peut affirmer, les seuls zéros de $\gamma_{+}(w)$ étant en $1,3,5, \ldots$, que les seules fonctions entières en $u$ parmi les $C_{a}(u, w), w \in \mathbb{C}$, sont obtenues pour $w \in\{ \pm 1, \pm 3, \pm 5, \ldots\}$ et que $C_{a}(u, w)$ est dans $K$ si et seulement si soit $\operatorname{Re}(w)<1 / 2$ soit $w \in\{1,3,5, \ldots\}$. Nous avons ainsi établi:

Lemme 1.2. La fonction $C_{a}(u, w)$ pour $u>0$ fixé est une fonction entière de $w$.

Lemme 1.3. Pour $w \in \mathbb{C}$ fixé $C_{a}(u, w)$ est une fonction analytique de $\left.\left.u \in \mathbb{C} \backslash\right]-\infty, 0\right]$. Elle est entière si et seulement si $w \in\{ \pm(1+2 j), j \in \mathbb{N}\}$. Elle est $O(1 / u)$ sur $[1, \infty[$, et cela uniformément par rapport à $w$ lorsque $|w|$ est borné. Pour $\operatorname{Re}(w)>0$, elle vaut $\gamma_{+}(w) u^{-w}-2 \int_{0}^{a} \cos (2 \pi u t) t^{w-1} d t$, et est donc $\gamma_{+}(w) u^{-w}+O(1)$ sur $\left.] 0,1\right]$ et cela uniformément par rapport à $w$ pour $\operatorname{Re}(w) \geq \epsilon>0$. Elle appartient à $K$ si et seulement si soit $\operatorname{Re}(w)<1 / 2$ soit $w \in\{1,3,5, \ldots\}$. On a $C_{a}(u, 1+2 j)=$ $-\mathcal{F}_{+}\left(\mathbf{1}_{t \leq a} t^{2 j}\right)(u)$ pour $j \in \mathbb{N}$.

ThÉORÈme 1.4. Soient $a>0, b>0$ fixés. Les transformées de Mellin $\widehat{f}(s)$ des fonctions $f \in K_{a, b}$ possèdent en $1,3,5, \ldots$ des zéros triviaux et ce sont leurs seuls zéros communs.

DÉmonstration. Nous donnons ici une approche directe qui ne fait pas appel aux résultats généraux de [13]. Le fait que $\widehat{f}(1+2 j)=0(j \in \mathbb{N})$ pour tout $f \in K_{a, b}$ se voit directement 
sur l'équation (1.1.). Pour tout nombre complexe $w$ et toute fonction $f \in K_{a, b}$ on a $\widehat{f}(w)=$ $\left(\mathcal{F}_{+}(f), \phi_{a, b}^{w}\right]=\left(f, \mathcal{F}_{+}\left(\phi_{a, b}^{w}\right)\right]$ avec $\phi_{a, b}^{w}(u)=\mathbf{1}_{u \geq b}(u) C_{a}(u, w)$. Donc si $w$ est un zéro commun à toutes les fonctions $\widehat{f}$ alors $\left.\left.\left.\left.\phi_{a, b}^{w} \in \mathcal{F}_{+}\left(L^{2}(] 0, a\right]\right)\right)+L^{2}(] 0, b\right]\right)$. Dans l'écriture (unique) correspondante $\phi_{a, b}^{w}=f_{a, b}+g_{a, b}$ la fonction $f_{a, b}(u)$ est une fonction entière de $u$ et elle est égale pour $u>b$ à $C_{a}(u, w)$ qui est analytique sur $\left.\left.\mathbb{C} \backslash\right]-\infty, 0\right]$. Elles sont donc égales et on en déduit que $C_{a}(u, w)$ appartient à $\left.\left.\mathcal{F}_{+}\left(L^{2}(] 0, a\right]\right)\right)$. En particulier elle est dans $K$ ce qui implique par ce qui précède soit $w \in\{1+2 j, j \in \mathbb{N}\}$ soit $\operatorname{Re}(w)<1 / 2$. Mais ce dernier cas est à exclure car alors $C_{a}(u, w)$ appartient à $\mathcal{F}_{+}\left(L^{2}([a, \infty[))\right.$ (et est non nulle).

L'équation (1.1) montre que $1,3,5, \ldots$ sont simples en tant que zéros communs aux fonctions $\widehat{f}(s)$ pour $f \in K_{a, b}$. Il existe une isométrie canonique entre $K_{a, b}$ et $K_{\lambda, \lambda}$ pour $\lambda^{2}=a b$ et nous supposerons dorénavant $a=b=\lambda>0$. Nous définissons des fonctions $D_{w, k}(t)$ sur $] 0, \infty[$ et des vecteurs $X_{w, k}^{\lambda}$ dans $K$ pour $w \in \mathbb{C}$ et $k \in \mathbb{N}$ de la manière suivante: $X_{w, k}^{\lambda}(t):=\mathbf{1}_{t \geq \lambda}(t) D_{w, k}(t)$ avec, pour $\operatorname{Re}(w)>1 / 2, D_{w, k}(t):=(\log (1 / t))^{k} t^{-w}$ et, pour $\operatorname{Re}(w) \leq 1 / 2, D_{w, k}(t):=\left(d^{k} / d^{k} w\right) C_{\lambda}(t, w)$. Pour tout $f \in K_{\lambda, \lambda}$ on a pour $\operatorname{Re}(w)>1 / 2\left(f, X_{w, k}^{\lambda}\right]=\left(d^{k} / d^{k} w\right) \widehat{f}(1-w)$ et pour $\operatorname{Re}(w) \leq 1 / 2$ on a $\left(f, X_{w, k}^{\lambda}\right]=\left(d^{k} / d^{k} w\right)\left(\gamma_{+}(w) \widehat{f}(1-w)\right)$. On notera en particulier la compensation entre les pôles de $\gamma_{+}(w)$ et les zéros triviaux de $\widehat{f}(1-w)$.

ThÉORÈme 1.5. Soit $\lambda>0$. Les projections orthogonales des vecteurs $X_{w, k}^{\lambda}$ sur $K_{\lambda, \lambda}$ sont linéairement indépendantes.

DÉmonstration. Supposons qu'une combinaison linéaire finie des $X_{w, k}^{\lambda}$ soit dans $K_{\lambda, \lambda}^{\perp}=$ $\left.\left.\left.\left.L^{2}(] 0, \lambda\right]\right)+\mathcal{F}_{+}\left(L^{2}(] 0, \lambda\right]\right)\right)$. Comme dans la démonstration précédente, la combinaison linéaire correspondante des $D_{w, k}(t)$ (qui est analytique en $t$ sur $] 0, \infty\left[\right.$ ) doit être dans $\left.\mathcal{F}_{+}\left(L^{2}(] 0, \lambda\right]\right)$ ). Elle ne peut être de carré intégrable au voisinage de l'origine que si il n'y a aucune contribution d'un couple $(k, w)$ avec $\operatorname{Re}(w)>1 / 2$ car alors $D_{w, k}(t)=(\log (1 / t))^{k} t^{-w}$. On élimine pour la même raison les couples $(w, k)$ vérifiant $\operatorname{Re}(w)=1 / 2$ car alors $D_{w, k}(t)$ a par le Lemme (1.3) une singularité dominante pour $t \rightarrow 0$ du type $(\log (1 / t))^{k} t^{-w}$. On remarque finalement pour $\operatorname{Re}(w)<1 / 2$ par l'équation (1.1) l'identité $\left(\mathcal{F}_{+}(f), X_{w, k}^{\lambda}\right]=\left(d^{k} / d^{k} w\right)(\widehat{f}(w))$ qui montre que $\mathcal{F}_{+}\left(X_{w, k}^{\lambda}\right)$ et $(-1)^{k} X_{1-w, k}$ ont la même projection sur $K_{\lambda, \lambda}$. On est donc ramené au cas précédemment éliminé. 


\section{Les espaces $H_{\Lambda}$ et les vecteurs $Z_{w, k}^{\Lambda}$}

Il est naturel pour les développements ultérieurs de travailler plutôt avec les fonctions $\widehat{f}(1-s)$, pour lesquelles les zéros triviaux sont situés en $0,-2,-4, \ldots$ Soit $H_{\Lambda}$ (pour $\Lambda>0$ ) l'espace des fonctions dans $K$ presque partout nulles pour $t>\Lambda$ et dont les images sous $\mathcal{G}:=I \mathcal{F}_{+} I$ sont presque partout nulles pour $t>\Lambda$. On a $H_{\Lambda}=I K_{\lambda, \lambda}$ avec $\lambda=1 / \Lambda$ et les transformées de Mellin sont les fonctions $\widehat{f}(1-s), f \in K_{\lambda, \lambda}$. L'unitaire $\mathcal{G}$ vérifie $\mathcal{G}^{2}=1$ et laisse stable $H_{\Lambda}$ qui se décompose donc comme une somme perpendiculaire $H_{\Lambda}^{+} \perp H_{\Lambda}^{-}$, les transformées de Mellin des fonctions invariantes sous $\mathcal{G}$ satisfaisant la même équation fonctionnelle que la fonction dzêta de Riemann, tandis que celles dans $H_{\Lambda}^{-}$la satisfont au signe près. Il est clair que les espaces $H_{\Lambda}$ forment une chaîne croissante d'espaces de Hilbert.

Proposition 2.1. On $a \bigcap H_{\Lambda}=\{0\}$ et $\overline{U H_{\Lambda}}=K$.

La première assertion est immédiate, et pour la deuxième on peut par exemple utiliser le fait que l'espace $\bigcup H_{\Lambda}$ est invariant sous les translations multiplicatives $D_{\theta}: f(t) \mapsto(1 / \sqrt{\theta}) f(t / \theta)$ $(0<\theta<\infty)$, puisque $D_{\theta}\left(H_{\Lambda}\right) \subset H_{\Lambda \theta}$ pour $\theta \geq 1$ et $D_{\theta}\left(H_{\Lambda}\right) \subset H_{\Lambda / \theta}$ pour $\theta \leq 1$. Or si $f(t)$ est une fonction quelconque non-nulle de $H_{1}$ alors les zéros de $\widehat{f}(s)$ sur la droite critique forment un ensemble de mesure de Lebesgue nulle et par un théorème de Wiener cela implique que les combinaisons linéaires de ses translatées multiplicatives sont denses dans $K$.

Au vu des zéros triviaux en $0,-2, \ldots$ on associe à $f \in H_{\Lambda}$ non plus simplement $\widehat{f}(s)$ mais la fonction entière $M(f)(s):=\pi^{-s / 2} \Gamma(s / 2) \widehat{f}(s)$. L'espace de Hilbert des fonctions entières $M(f)(s)$ pour $f \in H_{\Lambda}$ est un Espace de Sonine au sens de [13], [14] (ici, la droite critique joue le rôle de l'axe réel dans [13], [14]). On dispose des équations fonctionnelles $M(\mathcal{G}(f))(s)=M(f)(1-s)$.

Proposition 2.2. Pour tout nombre complexe $w$ et pour tout entier $k \geq 0$ l'évaluation $f \mapsto$ $M(f)^{(k)}(w)$ définit une forme linéaire continue sur $H_{\Lambda}$.

Cela découle des calculs précédant le Théorème (1.5) puisque ces formes linéaires s'expriment (selon un système triangulaire) en fonction des produits scalaires euclidiens avec les vecteurs $I\left(X_{w, k}^{\lambda}\right)$ $(\lambda=1 / \Lambda)$. Il existe donc un unique vecteur $Z_{w, k}^{\Lambda}$ dans $H_{\Lambda}$ tel que $\forall f \in H_{\Lambda} M(f)^{(k)}(w)=\left(f, Z_{w, k}^{\Lambda}\right]$. Le noyau reproduisant (analytique) $K^{\Lambda}(w, k ; z, l)=\left(Z_{w, k}^{\Lambda}, Z_{z, l}^{\Lambda}\right]$ est un objet important de l'Analyse pour lequel la théorie de De Branges donne une formule exacte à partir d'une certaine fonction $E_{\Lambda}(z)$ 
au sujet de laquelle nous souhaiterions pouvoir être plus explicite. Les vecteurs $Z_{w, k}^{\Lambda}$ sont reliés aux projections orthogonales des vecteurs $I\left(X_{w, k}^{\lambda}\right)(\lambda=1 / \Lambda)$ sur $H_{\Lambda}$ par un système triangulaire inversible et donc par le Théorème (1.5):

ThÉORÈme 2.3. Les vecteurs $Z_{w, k}^{\Lambda}$ pour $w \in \mathbb{C}, k \in \mathbb{N}$ sont linéairement indépendants (en particulier ils sont tous non nuls.)

\section{Les espaces $W_{\Lambda}$ et $H P_{\Lambda}$ pour $\Lambda>1$}

Soit $\Lambda>1$ et soit $\mathcal{V}_{\Lambda}$ l'espace vectoriel des fonctions infiniment différentiables et supportées par $[1 / \Lambda, \Lambda]$. La transformée de Mellin $\widehat{\phi}(s)$ d'une telle fonction $\phi$ est une fonction entière. Le produit $\zeta(s) \widehat{\phi}(s)$ est une fonction méromorphe, de carré intégrable sur la droite critique et qui est la transformée de Mellin au sens $L^{2}$ de la fonction $E(\phi)(u)=\sum_{n \geq 1} \phi(n u)-\left(\int_{0}^{\infty} \phi(t) d t\right) / u([5]$.) Soit $D=u\left(d^{2} / d^{2} u\right) u$ l'opérateur différentiel invariant de multiplicateur spectral $s(s-1)$. Les fonctions de $\mathcal{V}_{\Lambda}$ dans l'image de $D$ sont exactement celles vérifiant $\widehat{\phi}(0)=\widehat{\phi}(1)=0$. On désignera par $W_{\Lambda}$ l'adhérence dans $K$ des fonctions $E(\phi)$ pour $\phi \in D\left(\mathcal{V}_{\Lambda}\right)$.

ThÉORÈme 3.1. Soit $\Lambda>1$. On a $W_{\Lambda} \subset H_{\Lambda}$. Un vecteur $Z_{w, k}^{\Lambda}$ est perpendiculaire à $W_{\Lambda}$ si et seulement si w est un zéro non-trivial $\rho$ de la fonction dzêta de Riemann et $0 \leq k<m_{\rho}\left(m_{\rho}=\right.$ multiplicité de $\rho$.)

DÉmonstration. On observe pour $\phi \in D\left(\mathcal{V}_{\Lambda}\right)$ que $E(\phi)$ a son support dans $\left.] 0, \Lambda\right]$. De plus $I(\phi)$ est aussi dans $D\left(\mathcal{V}_{\Lambda}\right)$ et donc $E I(\phi)$ a également son support dans $\left.] 0, \Lambda\right]$. La transformée de Mellin de $E(\phi)$ est $\zeta(s) \widehat{\phi}(s)$ et son image sous $\mathcal{F}_{+} I$ est $\gamma_{+}(s) \zeta(s) \widehat{\phi}(s)=\zeta(1-s) \widehat{\phi}(s)$. Donc $\mathcal{G}(E(\phi))=$ $I \mathcal{F}_{+} I(E(\phi))$ a comme transformée de Mellin $\zeta(s) \widehat{\phi}(1-s)$ autrement $\operatorname{dit} \mathcal{G}(E(\phi))=E(I(\phi))$. Il existe

des fonctions $\widehat{\phi}(s)$ avec $\phi \in \mathcal{V}_{\Lambda}$ prenant des valeurs et des dérivées quelconques en un nombre fini de nombres complexes fixés à l'avance et avec cela le Théorème est démontré. •

Définition 3.2. Soit $\Lambda>1$. On désigne par $H P_{\Lambda}$ le complément orthogonal de $W_{\Lambda}$ dans $H_{\Lambda}$.

On comparera à l'approche de Connes dans [11], [12]. On a donc

$$
K=\left(L^{2}((\Lambda, \infty), d t)+\mathcal{G} L^{2}((\Lambda, \infty), d t)\right) \perp W_{\Lambda} \perp H P_{\Lambda}
$$

On peut traiter de manière analogue le cas d'une série $L(s, \chi)$ de Dirichlet pour les caractères primitifs et pairs. Il sera alors inutile de se restreindre à $D\left(V_{\Lambda}\right)$ et il faudra tenir compte du 
conducteur $q>1$. Pour une série associée à un caractère impair la construction utilisera non plus $\mathcal{F}_{+}$mais la transformation en sinus $\mathcal{F}_{-}$.

\section{Conclusion}

Dans la continuation de [4], [5], nous avons montré que la théorie (appartenant à l'Analyse) de collision (scattering) pour la transformation de Fourier a des aspects arithmétiques en associant de manière intrinsèque un certain quotient $H P_{\Lambda}$ à la fonction dzêta de Riemann (pour $\Lambda>1$ ) et en montrant qu'il est lié à ses zéros non-triviaux (et à leurs multiplicités éventuelles; ceci est dans la continuation de [10].) Il serait intéressant de mieux comprendre ces constructions en termes opératoriels ([6],[7],[8].) L'étude de $\Lambda \rightarrow 0$, de $\Lambda \rightarrow \infty$, et de $\Lambda=1$ est un problème de groupe de renormalisation ([16]) qui semble relié à celui de la nature de la fonction dzêta ([3].) 


\section{Références bibliographiques}

[1] Báez-Duarte L., A class of invariant unitary operators, Adv. in Maths. 144 (1999), 1-12.

[2] Báez-Duarte L., Balazard M., Landreau B. et Saias E., Notes sur la fonction $\zeta$ de Riemann 3, Adv. in Maths. 149 (2000), 130-144.

[3] Blake W., The Explicit Formula and a propagator, 21 p., http://fr.arXiv.org/abs/math/9809119

[4] Burnol J.-F., Scattering on the p-adic field and a trace formula, Int. Math. Res. Not. 2000:2 (2000), 57-70.

[5] - An adelic causality problem related to abelian L-functions, J. Number Theory 87 (2001), no. 2, 253-269.

[6] The Explicit Formula and the conductor operator, 28p., http://fr.arXiv.org/abs/math/9902080

[7] — Sur les Formules Explicites I: analyse invariante, C. R. Acad. Sci. Paris 331 (2000), série I, 423-428.

[8] _ Quaternionic gamma functions and their logarithmic derivatives as spectral functions, Math. Res. Lett. 8 (2001), no. 1-2, 209-224.

[9] — A note on Nyman's equivalent formulation for the Riemann Hypothesis, 4p., Cont. Math. Ser., à paraître.

[10] _ A lower bound in an approximation problem involving the zeros of the Riemann zeta function, 17p., mars 2001. Soumis à Adv. in Maths. http://fr.arXiv.org/abs/math/0103058

[11] Connes A., Formule de trace en géométrie non-commutative et hypothèse de Riemann, C. R. Acad. Sci. Paris 323 (1996), série I, 1231-1236.

[12] - Trace formula in non-commutative Geometry and the zeros of the Riemann zeta function, Selecta Math. (N.S.) 5 (1999), no. 1, 29-106.

[13] De Branges L., Espaces Hilbertiens de Fonctions Entières, Masson et Cie, Paris (1972) (trad. de Hilbert spaces of entire functions Prentice Hall Inc., Englewood Cliffs, 1968.)

[14] — A conjecture which implies the Riemann hypothesis, J. Funct. Anal. 121 (1994), no. 1, 117-184.

[15] Dym H., McKean H.P., Fourier Series and Integrals, Academic Press, 1972.

[16] Wilson K.G., The renormalization group and critical phenomena, Rev. Mod. Phys. 55 (1983), 583-600. 